hearts received 3 cycles of 2 min hypothermic perfusion $\left(26^{\circ} \mathrm{C}\right)$ interspersed by 3 min normothermic perfusion $\left(37^{\circ} \mathrm{C}\right)$. Other hearts received a single 6 min hypothermic perfusion (SHP) before ischaemia. During reperfusion, TP improved hemodynamic recovery, decreased arrhythmias, reduced lactate dehydrogenase release and mitochondrial permeability transition pore opening more than IP or SHP. Both TP and IP were blocked by pretreatment with $10 \mu \mathrm{M}$ chelerythrine (PKC inhibitor) and $300 \mu \mathrm{M}$ mercapto-propionyl glycine (free radical scavenger). $10 \mu \mathrm{M}$ Compound C (AMPK inhibitor) partially blocked cardioprotection by TP but not IP suggesting that free radicals, PKC and AMPK may mediate the effects of TP.

Keywords: Ischemia/reperfusion; Free radicals; Cardioprotection

doi:10.1016/j.yjmcc.2007.03.544

\section{Novel pharmacological compounds as triggers of ischemic preconditioning in vivo}

Ioanna Andreadou ${ }^{1,2}$, Theano Fotopoulou ${ }^{1,3}$, Maria Koufaki ${ }^{3}$, Andrew Tsotinis ${ }^{1}$, Anastasia Zoga ${ }^{2}$, Vassilis Gizas ${ }^{2}$, Efstathios Iliodromitis ${ }^{2}$, Dimitrios Th Kremastinos ${ }^{2} .{ }^{1}$ School of Pharmacy, University of Athens, Greece. ${ }^{2} \mathrm{~B}$ ' University Dept. of Cardiology, Medical School, Attikon General Hospital, University of Athens, Greece. ${ }^{3}$ National Hellenic Research Foundation, Athens, Greece

In the present study we assessed the cardioprotective effect of a series of new compounds, bearing aromatic heterocycles, such as indole, quinoline and adenine and the pharmacophoric nitroxy ester group. Rabbits were divided into 4 groups and subjected to $30 \mathrm{~min}$ ischemia and $3 \mathrm{~h}$ reperfusion with the following prior interventions: no intervention, administration of the indole analogue or quinoline analogue or N6, N9-substituted adenine analogue in a total dose of $4 \mathrm{mg}, 40 \mathrm{~min}$ and $1 \mathrm{~min}$ before sustained ischemia. Infarct size was determined and blood samples were taken for malondialdehyde (MDA) assessment as an index of lipid peroxidation. Infarct size reduced in all treated groups $(20.5 \pm 5.2 \%, 22.4 \pm 4.7 \%, 19.8 \pm 3.1 \%$ vs. $47.4 \pm 2.6 \%$ in control, $P<0.05)$. Combined treatment with the indole derivative and the mito $\mathrm{K}_{\text {ATP }}$ blocker 5-hydroxydecanoic acid (5-HD) abolishes the reduction of infarct size while combined adenine analogue and 5-HD does not block the reduction. All the tested compounds reduced the level of MDA at the 20th min of reperfusion $(P<0.05)$, while it was significantly elevated at the 20 th min of ischemia in groups treated with the indole and the quinoline compared to baseline. We conclude that the new compounds trigger pharmacologically a preconditioning like effect and reduce the infarct size.

Keywords: Cardioprotection; Infarct size; Preconditioning

doi:10.1016/j.yjmcc.2007.03.545
Lipid raft and map kinase interaction play a key role in angiotensin-II preconditioning of the heart

Manika Das, Samarjit Das, Dipak K. Das. University of Connecticut School of Medicine, 263 Farmington Avenue, Farmington, CT-06030, USA

Previous studies showed that angiotensin II [Ang II ] could precondition $[\mathrm{PC}]$ heart by modulating MAP kinase signaling. Since lipid raft can modulate MAP kinase signaling, we hypothesized that lipid raft could play a role in Ang II PC. A group of rat hearts was treated with Ang II in absence and presence of a NADPH oxidase inhibitor, apocyanin or a cell permeable ROS scavenger, $N$-acetylcysteine (NAC). Ang II improved post-ischemic ventricular recovery, reduced myocardial infraction and cardiomyocyte apoptosis. Both apocyanin and NAC abolished cardioprotection of Ang II PC. In Ang II treated heart, there was decreased association of anti-death signaling components (p38MAPK $\beta$, ERK1/2) with caveolin while there was increased association of death signaling components (p38MAPK $\alpha$, JNK) indicating reduced amount of death signaling components and increased amount of anti-death signaling components were available to the Ang II treated heart to generate survival signal. In contrast, in $\mathrm{I} / \mathrm{R}$ heart, there was an increased association of anti-death signaling components with caveolin while there was reduced association of death signaling components indicating increased amount of death signaling components were available to $I / R$ hearts to generate death signal. The survival signal was further confirmed by increased phosphorylation of AKT and enhanced induction of expression of BCL-2 during Ang II precondition and its reversal with $\mathrm{I} / \mathrm{R}, \mathrm{NAC}$ and apocyanin treated hearts. These results indicate a unique MAPK signaling in Ang II heart working through lipid raft.

Keywords: Precondition; Ang II; Caveolin

doi:10.1016/j.yjmcc.2007.03.546

\section{PKC enhances adenosine $A_{2 b}$ receptor sensitivity in preconditioned rabbit hearts}

Atsushi Kuno, Lin Cui, Michael V. Cohen, James M. Downey. University of South Alabama, Mobile, AL, USA

Activation of $\mathrm{PKC}$ is central to the protection of ischemic preconditioning (IPC), but PKC's actual role in this process is unknown. Protection from IPC in isolated rabbit hearts is dependent on PKC activity during the first minutes of reperfusion and direct activation of PKC with phorbol 12 myristate 13acetate (PMA, $0.05 \mathrm{nM}$ ) for 6 min starting 1 min before reperfusion duplicates IPC's protection. Either chelerythrine $(2.8 \mu \mathrm{M})$, a PKC inhibitor, MRS1754 $(20 \mathrm{nM})$ an adenosine $\mathrm{A}_{2 \mathrm{~b}}$ receptor $\left(\mathrm{A}_{2 \mathrm{~b}} \mathrm{R}\right)$ antagonist, or U0126 $(0.5 \mu \mathrm{M})$, an ERK antagonist, abolishes protection from PMA. We tested whether PKC activation might be altering sensitivity to adenosine. Intracoronary $5 '$ - $(N$-ethylcarboxa-mido) adenosine (NECA) $(10 \mathrm{nM})$, an adenosine receptor agonist, nearly doubled Akt and ERK 